\title{
The prognostic roles of mRNAs of the exosomes derived from bone marrow stromal cells in common malignancies: a bioinformatic study
}

This article was published in the following Dove Press journal: OncoTargets and Therapy

Fei Zhao

Yan-Qiu Yu

Department of Pathophysiology, College of Basic Medicine Science, China Medical University, Shenyang, Liaoning, China
Correspondence: Yan-Qiu Yu Department of Pathophysiology, College of Basic Medicine Science, China Medical University, Shenyang, Liaoning, II0I22, China

Tel +86 I 3386887215

Email yqyu@cmu.edu.cn
Background: The inclusion of exosomes enters the recipient cells by means of endocytosis or direct fusion for information exchange between cells and cells. The inclusion of BMSCs-exo helps to guide the diagnosis and prognosis of cancer, especially cancer.

Purpose: This research was to systematically elucidate the prognostic value of mRNAs of the exosomes derived from bone marrow stromal cells (BMSCs) in common malignant neoplasms, such as breast cancer, ovarian cancer, lung cancer, and gastric cancer.

Methods: Gene expression data (GSE78235) for the exosomes derived from BMSCs were extracted from the Gene Expression Omnibus database. Firstly, the differentially expressed genes were detected by comparing the RNA expression from exosomes derived from BMSCs between four tumor patients and two healthy controls using the limma package. Subsequently, functional enrichment analysis, including Gene Ontology and Kyoto Encyclopedia of Genes and Genomes of differentially expressed genes, was performed using the Functional Enrichment analysis tool (FunRich v3.1.3) software followed by the construction of a protein-protein interaction (PPI) network via STRING v3.6.0. Molecular Complex Detection was used to screen the hub proteins by setting up the following threshold score $\geq 4$ and nodes $\geq 10$. Cytoscape v3.6.1 was used to for visualizing PPI network. Finally, Kaplan-Meier analysis for hub proteins was performed by Kaplan-Meier plotter online platform.

Results: A total of 386 genes originating from the exosomes derived from BMSCs were identifed as statistically signifcant $(P<0.05, \mathrm{FDR}<0.05)$, which consisted of 150 upregulated genes and 236 downregulated genes. Also, 32 pathways were identifed as signifcant $(P<0.05$, FDR $<0.05)$. The PPI network of exosomes derived from BMSC proteins included 100 protein nodes with 579 interaction edges. The hub proteins, including PODN, ZNF521, and CFI, which interacted with ten or more other proteins, were indicated as the hub proteins of PPN of exosomes derived from BMSCs.

Conclusion: Taken together, our findings revealed the prognostic roles of mRNAs of exosomes derived from BMSCs and provided implications for targeted therapy for common malignant neoplasms. However, further studies require large samples and experimental verification.

Keywords: bioinformatics, BMSCs, clinical outcome, exosome, malignant neoplasms

\section{Introduction}

Mesenchymal stromal cells (MSCs), also known as mesenchymal stem cells, have the potential for self-renewal and multidirectional differentiation. ${ }^{1}$ Considering the source, MSCs can be isolated from multiple tissues, such as bone marrow, umbilical cord, blood, placenta, and adipose tissue. ${ }^{2-5}$ It was reported that bone marrow mesenchymal cells (BMSCs) that originated from the bone marrow can secrete a diverse variety of bioactive factors in an autocrine and/or paracrine way, which was expected to become 
a non-cellular therapy for the treatment of various diseases, especially tumors. ${ }^{6-9}$ Of them, an important paracrine factor of BMSCs is the exosome, which is a lipid membrane wrapped vesicle with a diameter of 40-200 nm. Exosome is abundant in miRNA, mRNA, lipids, and proteins. It has been reported that exosomes enter the recipient cells by endocytosis, or directly fuse with the recipient cells to exchange information between cells. ${ }^{10-13}$ Exosomes derived from BMSCs have been able to treat proangiogenic, cardiovascular disease, liver disease, lung injury, and kidney injury. ${ }^{14-18}$ Indeed, there are two opposite viewpoints on the role of exosome in the treatment of tumors. Some studies have suggested that exosomes have a strong antitumor effect, while other studies have shown that exosomes derived from MSCs can promote the growth and metastasis of cancer cells. Disappointingly, the mechanism of antitumor effect of MSC-derived exosomes remains unclear. ${ }^{19}$

Malignant tumors cause an extremely large burden around the world, second only to cardiovascular disease..$^{20,21}$ Despite using combinations of various strategies of diagnosis and treatment, the prognosis of cancer patients remains poor. ${ }^{22}$ Therefore, it is necessary to explore multiple effective early diagnostic and prognostic markers. Recently, it has been reported that exosomes are involved in cancer development, progression, and resistance; therefore, they may be used as multiple targets for the development of a diagnostic tool for early detection of cancer. ${ }^{23-26}$ Along with the advances in microarray and life molecules sequencing technology, highthroughput research methods are widely used in biomedicine. High-throughput and corresponding bioinformatics analysis of exosomes is helpful to guide the diagnosis and prognosis of diseases, especially cancer. ${ }^{27,28}$ In this study, our goal was to identify the prognostic roles of differentially expressed genes originating from exosomes derived from BMSCs in common cancers, which provide new diagnostic and therapeutic targets for cancers.

\section{Materials and methods}

\section{Microarray data extraction and identifying} differentially expressed genes

We downloaded the gene expression profile (GSE78235) from the public availability repository Gene Expression Omnibus (GEO; https://www.ncbi.nlm.nih.gov/geo/) database. GEO2R online tool allows researchers to carry out R-based analysis of GEO data to identify differentially expressed genes (DEGs). Subsequently, the Database for Annotation, Visualization, and Integrated Discovery (https:// david.ncifcrf.gov/) v6.8 was used to perform re-annotation for DEGs. A gene is defined as a DEG between the tumor sample and the normal control sample when the false discovery rate
(FDR) is $<0.05(\mathrm{FDR} \leq 0.05)$ and the fold change $(\mathrm{FC})$ is at least two times higher or lower $(|\log 2 \mathrm{FC}| \geq 1)$.

\section{Functional enrichment analysis for DEGs}

Functional enrichment analysis was performed for DEGs to give a functional overview of the DEGs by calculating the whole significance of the gene expression. Gene ontology (GO) comprises molecular function, cellular component, and biological process. Kyoto Encyclopedia of Genes and Genomes (KEGG) was used to clarify how DEGs perform function through a certain path. We selected Functional Enrichment analysis tool (FunRich v3.1.3), which is designed to handle a variety of gene/protein datasets irrespective of the organism, to perform pathway enrichment analysis. FDR $<0.05$ was set as the cut-off criterion.

\section{Construction of protein-protein interaction network for DEGs and identification of hub proteins}

Protein-protein interaction (PPI) was used to analyze the interrelationship between differentially expressed proteins, and further elucidate the mechanisms of genes playing important roles in physiological and pathological conditions. The STRING database (http://string-db.org) helps to provide a critical assessment and integration of protein-protein interactions, including direct (physical) and indirect (functional) associations. In this study, we used STRING database v10.5 to construct PPI network for DEGs. The parameters were set as follows: meaning of network edges: confidence; minimum required interaction score: medium confidence (0.400). Then, Cytoscape software v3.6.0 was used to visualize the PPI network. At last, Cytoscape software plug-in Molecular Complex Detection was used to screen hub proteins within the PPI network.

\section{Kaplan-Meier (KM) plot analysis for hub proteins}

We ran a KM analysis for three hub genes to characterize the association of gene expression and corresponding clinical outcome in common malignant tumor. $\mathrm{KM}$ analysis is available on the website http://www.kmplot.com/. The KM plotter is capable to assess the effect of any gene or gene combination on survival in breast, ovarian, lung, gastric, liver cancer patients using over 30,000 samples measured using gene chips or RNA-seq.

\section{Results \\ Identification of DEGs from BMSCs- derived exosomes}

Based on GEO2R online platform, using FDR $<0.05$ and $(|\log 2 \mathrm{FC}| \geq 1)$ as the cut-offs, 386 genes were identified to 
be differentially expressed in the tumor compared with the normal control samples, which included 150 upregulated genes and 236 downregulated genes.

\section{GO enrichment analysis}

The eight important terms are obtained by GO enrichment analysis in the software FunRich as shown in Figures 1A-C.) The genes were enriched in biological processes of response to signal transduction, cell communication, cell growth and maintenance, and energy pathways metabolism. As for the molecular function, these genes showed enrichment in GTPase activity, chaperone activity, catalytic activity, calcium ion binding, and receptor
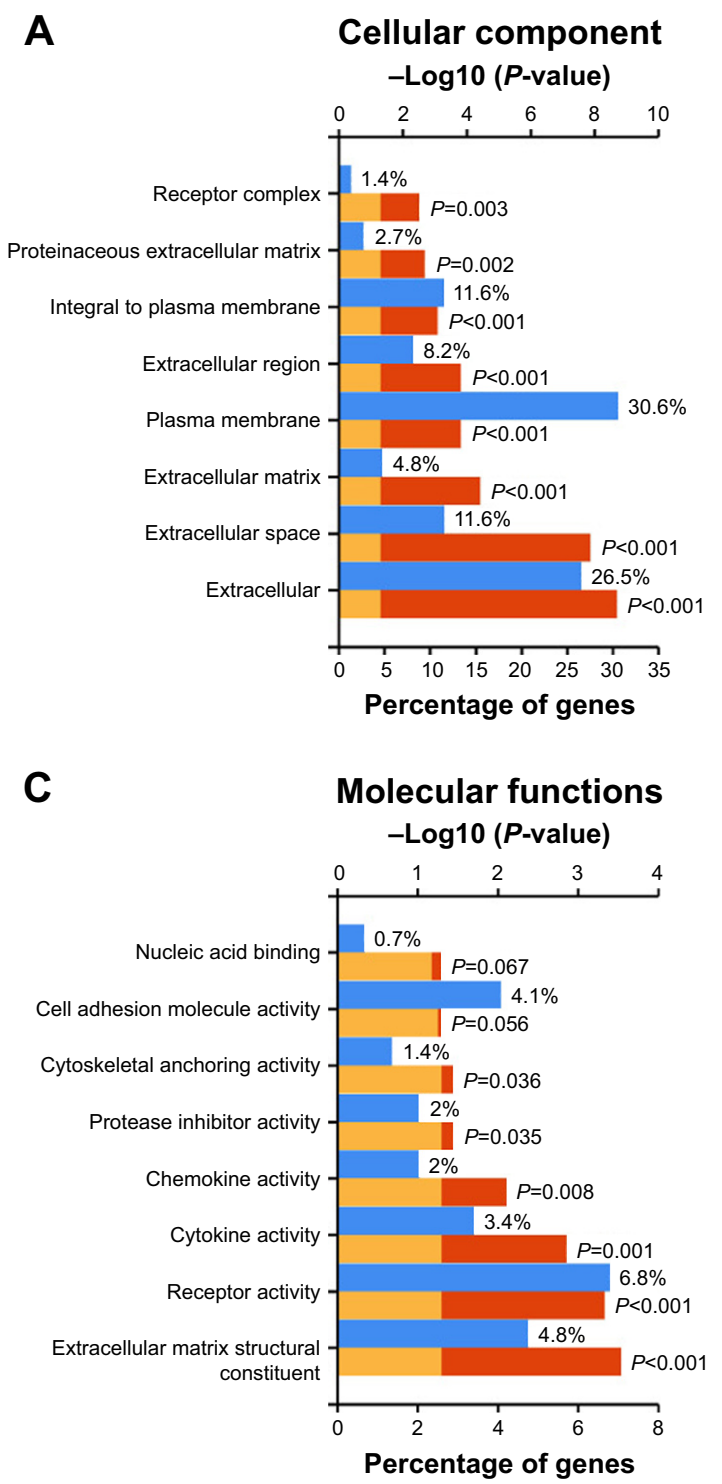

signaling complex scaffold activity. Besides, the cell component indicated enrichment predominantly at exosomes, cytoplasm, lysosome, nucleus, and plasma membrane.

\section{KEGG pathway analysis}

KEGG pathway enrichment analysis suggested that the genes were significantly enriched in pathways including vascular endothelial growth factor (VEGF) and VEGF receptor (VEGFR) signaling network, thrombin/protease-activated receptor pathway, PAR1-mediated thrombin signaling events, signaling events mediated by VEGFR1 and VEGFR2, integrin family cell surface interactions, IL5-mediated
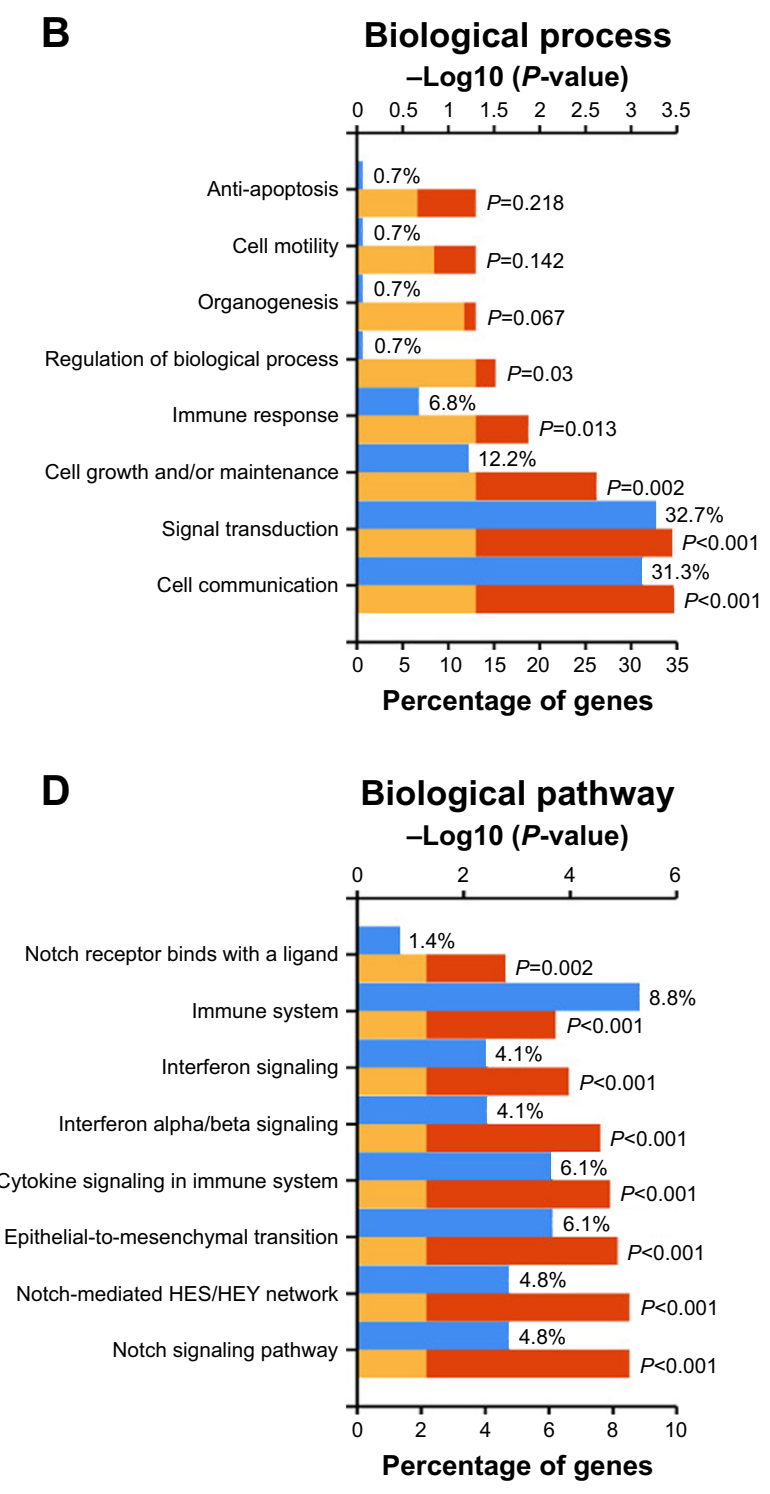

Percentage of gene $P=0.05$ reference $P$-value

Figure I The eight important terms are obtained by GO enrichment analysis in the software FunRich.

Notes: (A-C) The top eight terms of cell components, biological processes and molecular functions of GO enrichment analysis for DEGs, respectively. (D) The top eight terms of biological pathway of KEGG enrichment analysis for DEGs.

Abbreviations: DEGs, differentially expressed genes; GO, Gene Ontology; KEGG, Kyoto Encyclopedia of Genes and Genomes; HES/HEY, hairy/enhancer of split. 
signaling events, beta1 integrin cell surface interactions, syndecan-1-mediated signaling events, plasma membrane estrogen receptor signaling, and proteoglycan-mediated signaling events (Figure 1D).

\section{PPI network construction and hub module analysis}

We constructed a network for DEGs from the exosomes derived from BMSCs that included 579 interaction edges and 150 proteins nodes (Figure 2A). Next, we obtained four key modules by means of Cytoscape plug-in Molecular Complex Detection. At last, the three proteins that interacted with at least ten other proteins $(P<0.05$, FDR $<0.05$ ), which included PODN, ZNF521, and CFI, were identified as the hub proteins from four key modules (Figure 2B-E).

\section{Survival analysis of hub proteins}

We ran a KM plot analysis for three genes to visualize the association of hub genes expression and match the clinical outcome in breast cancer, ovarian cancer, lung cancer, and gastric cancer. The upregulation of PODN, ZNF521, and CFI indicated good survival in breast cancer (Figure 3A-C). Downregulation of PODN and ZNF521 indicated a good outcome in ovarian cancer (Figure 3D and E), while the upregulation of CFI indicated a good survival in ovarian cancer (Figure 3F). The downregulation of PODN, ZNF521, and CFI indicated good survival in lung cancer Figure $3 \mathrm{G}-\mathrm{I}$ ), while the upregulation of CFI indicated a good survival in gastric cancer (Figure 3L).

\section{Discussion}

Malignant tumor is a major public health problem that threatens human health and is second only to cardiovascular disease. Unfortunately, tumors lack early diagnostic and prognostic markers with a high sensitivity and specificity. Our study focuses on the critical role of mRNA of exosome derived from BMSCs. In our study, by extracting the gene expression information from GEO database, we obtained 386 DEGs, including 150 upregulated genes and 236 downregulated genes from the exosome derived from BMSCs. Next, we uncovered that those DEGs were mainly involved in proliferation and angiogenesis, such as VEGF and VEGFR signaling pathway. By constructing PPI network and identifying the hub genes, we obtained three hub proteins, including PODN, ZNF521, and CFI. These three genes are closely related to the clinical prognosis of common malignant tumors, including breast cancer, ovarian cancer, lung cancer, and gastric cancer, based on
KM online platform. In addition, three hub proteins have been reported less in the context of BMSCs. Moreover, the multiple roles of the three genes in common malignant neoplasm were also less studied. As illustrated in Figure 3, the upregulation of PODN, ZNF521, and CFI indicated good survival in breast cancer (Figure $3 \mathrm{~A}-\mathrm{C}$ ) and lung cancer (Figure 3G-I), compared with the downregulation of three hub genes. Downregulation of PODN and ZNF521 indicated a good outcome in ovarian cancer (Figure 3D and E), while the upregulation of CFI indicated a good survival in ovarian cancer (Figure 3F). Besides, the downregulation of PODN and ZNF521 indicated a good outcome in gastric cancer (Figure 3J and $\mathrm{K}$ ), while the upregulation of CFI indicated a good survival in gastric cancer (Figure 3L). As we know, malignant tumor is a group of diseases with high heterology. Moreover, a gene whether it is a tumor promoter or inhibitor depends on the microenvironment of the tumor.

However, we have noticed that the upregulation of PODN, ZNF521, and CFI indicated good survival in breast cancer (Figure 3A-C) and lung cancer (Figure 3G-I), compared with the downregulation of three hub genes. We make a bold assumption: the three hub genes may be used in the clinic for breast and lung cancers.

PODN was a seed gene in the first key modules. PODN, which is an important component of the extracellular matrix protein, belongs to the small leucine-rich repeat protein family. ${ }^{29}$ It was reported that PODN may bind type 1 collagen, thereby reducing the cell growth and migration in kidney. It is unfortunate that no literature has reported the role of PODN in tumors. ZNF521 was a seed gene in the second modules. ZNF521 is a zinc finger protein that consists almost entirely of $30 \mathrm{C} 2 \mathrm{H} 2$ Kruppel-like zinc fingers. Moreover, ZNF521 has been characterized as a potent inhibitor of EBF1 and is emerging as a potentially relevant contributor to the development of B-cell leukemias. ${ }^{31,32}$ ZNF521 contributes to clonogenic growth, migration, and tumorigenicity in medulloblastoma cells through the recruitment of the NuRD complex. ${ }^{30}$ Furthermore, ZNF521 sustains the differentiation block in myeloid-lymphoid leukemia-rearranged acute myeloid leukemia. CFI was a seed gene in the fourth modules. CFI is a serum protease that inhibits all complement pathways. The common variant rs10033900 near the CFI gene is associated with age-related macular degeneration risk in Han Chinese population. Based on the gene expression profile, we found that upregulated CFI indicates good prognosis, while Okroj et al suggested that high expression of CFI is associated with poor prognosis and recurrence in breast cancer. ${ }^{33}$ 


\section{A}

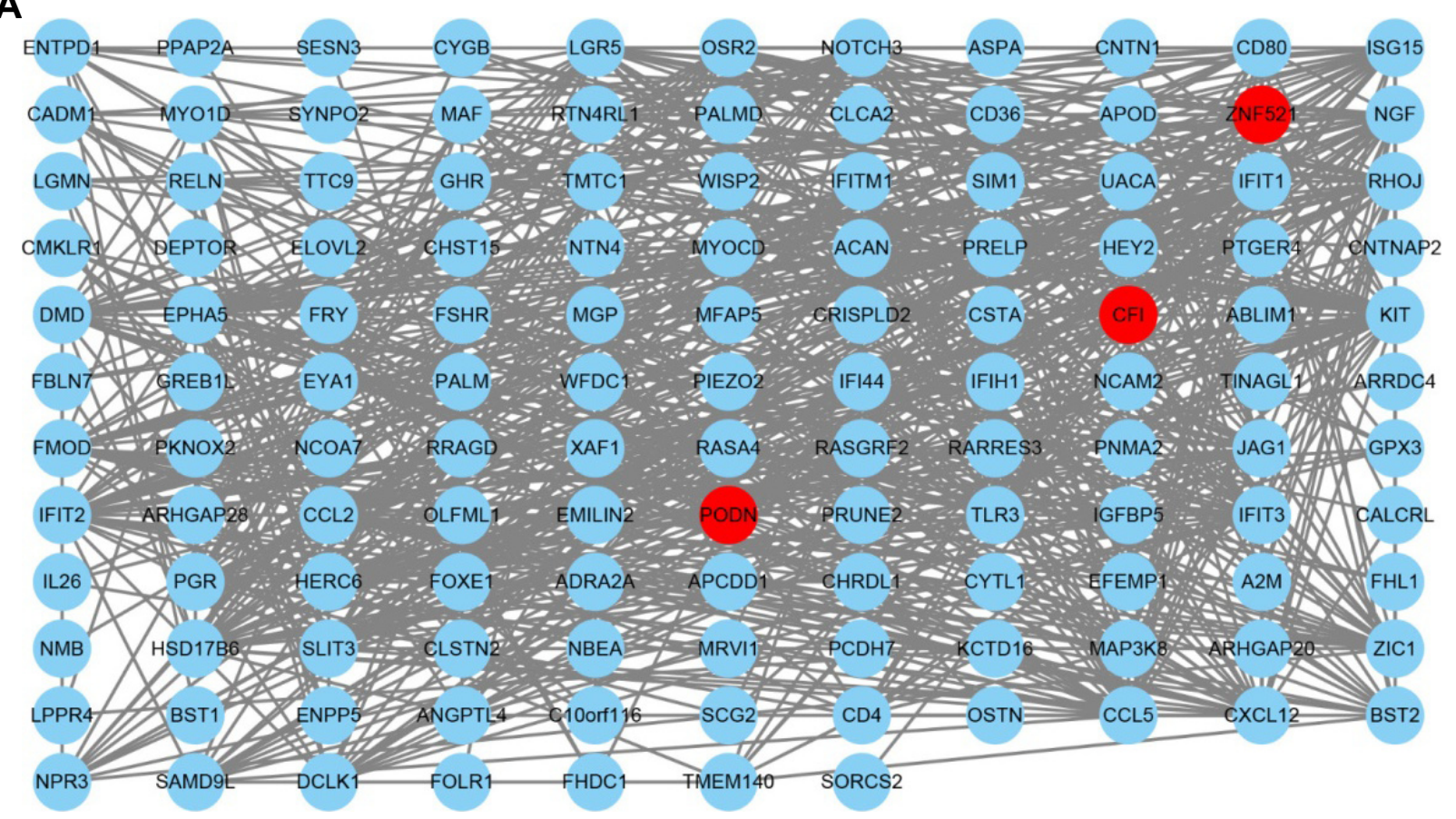

B

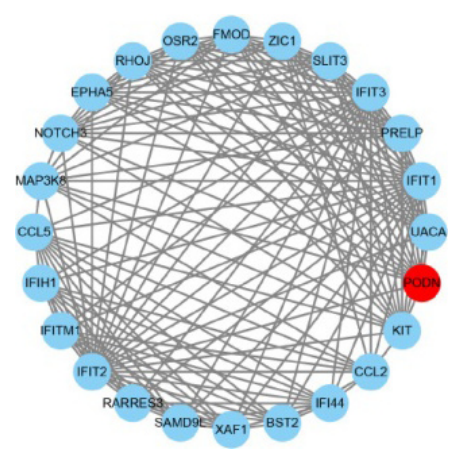

D

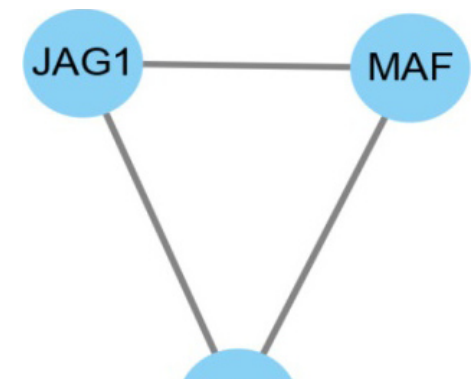

TLR3
C

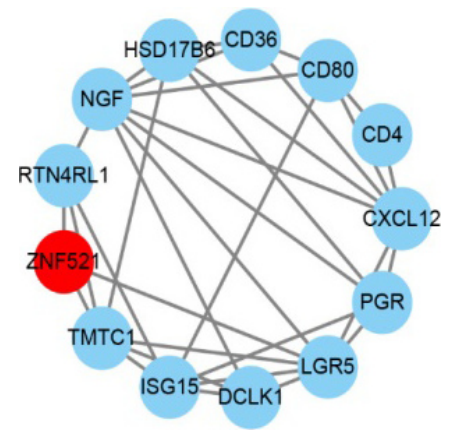

$\mathbf{E}$

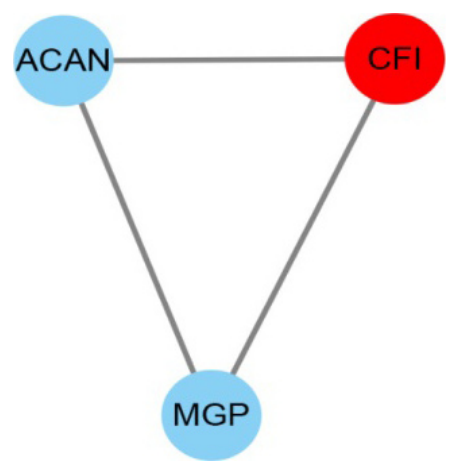

Figure 2 Through PPi network construction and hub module analysis identifies four central modules of key proteins.

Notes: (A) Protein-protein interaction network of the selected DEGs. (B-E) The four key gene modules calculated by MCODE software from the protein-protein interaction network. The red circle stands for the hub genes/proteins.

Abbreviations: DEGs, differentially expressed genes; MCODE, Molecular Complex Detection.

In our study, we systematically characterized DEGs from the exosomes derived from BMSCs and identified certain hub proteins and their PPI networks. Taken together, our findings suggested that three genes may be potential prognostic markers in common malignant tumors. However, the study results were obtained based on bioinformatics, which only previously considered datasets. However, further studies are required with large samples and for experimental verification. 
A

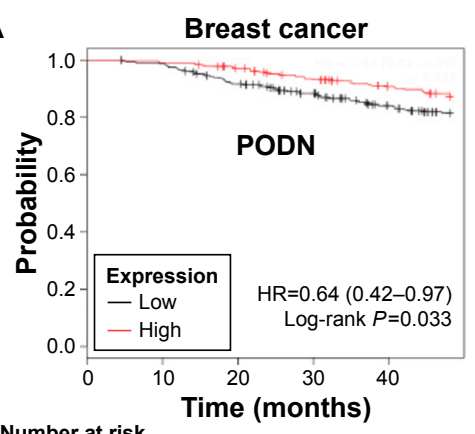

Number at risk

$\begin{array}{llllll}\text { Low } & 315 & 310 & 284 & 259 & 228\end{array}$

$\begin{array}{llllll}\text { High } & 311 & 308 & 297 & 280 & 263\end{array}$

D

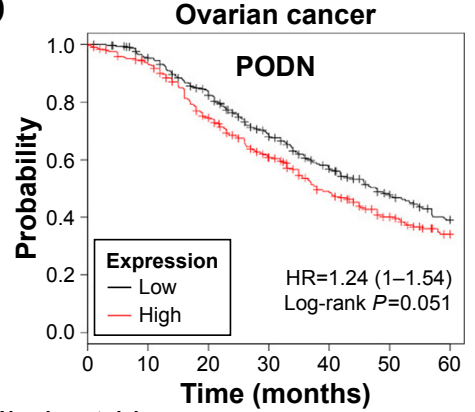

Number at risk

$\begin{array}{llllllll}\text { Low } & 332 & 305 & 257 & 186 & 136 & 93 & 70\end{array}$ $\begin{array}{llllllll}\text { High } & 323 & 287 & 218 & 157 & 106 & 73 & 51\end{array}$

G

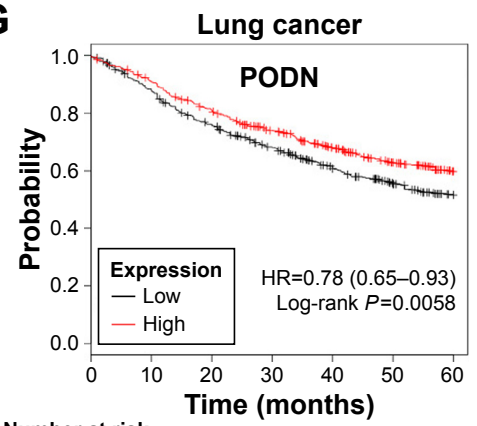

Number at risk

$\begin{array}{llllllll}\text { Low } & 572 & 496 & 422 & 365 & 305 & 258 & 213\end{array}$ $\begin{array}{llllllll}\text { High } & 573 & 517 & 456 & 397 & 342 & 288 & 232\end{array}$

$J$

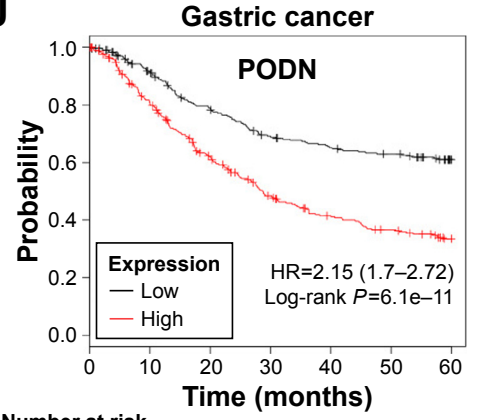

Number at risk

$\begin{array}{llllllll}\text { Low } & 301 & 260 & 220 & 190 & 117 & 169 & 143\end{array}$

$\begin{array}{llllllll}\text { High } & 330 & 253 & 183 & 136 & 111 & 96 & 79\end{array}$
B

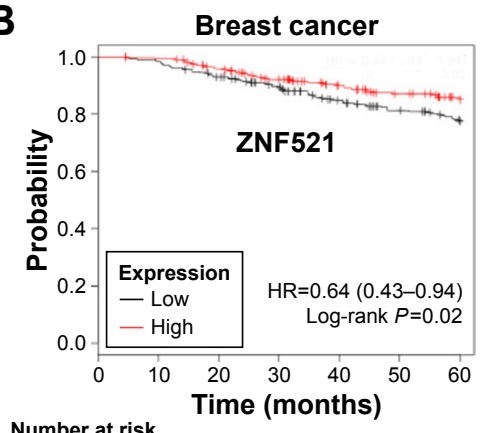

Number at risk

$\begin{array}{llllllll}\text { Low } & 313 & 308 & 288 & 268 & 241 & 223 & 207\end{array}$ $\begin{array}{llllllll}\text { High } & 313 & 310 & 293 & 271 & 250 & 231 & 213\end{array}$

E

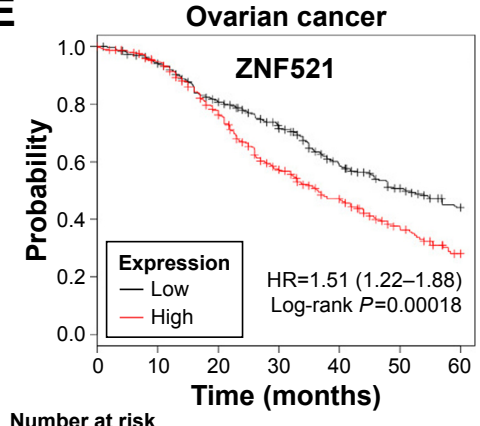

$\begin{array}{llllllll}\text { Low } & 333 & 301 & 247 & 196 & 141 & 106 & 84\end{array}$

$\begin{array}{llllllll}\text { High } & 322 & 291 & 228 & 147 & 101 & 60 & 37\end{array}$

H

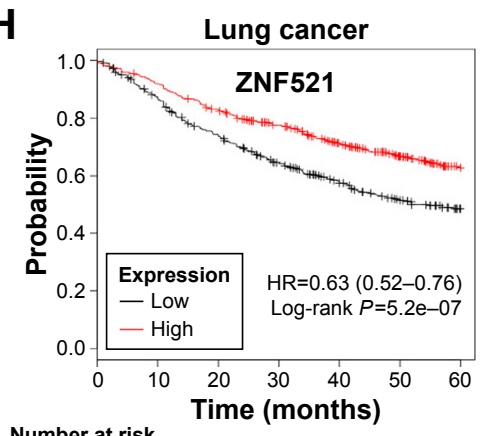

Number at risk

Time (months)

$\begin{array}{llllllll}\text { Low } & 573 & 489 & 409 & 344 & 288 & 243 & 199\end{array}$

$\begin{array}{llllllll}\text { High } & 572 & 524 & 469 & 418 & 359 & 303 & 246\end{array}$

K

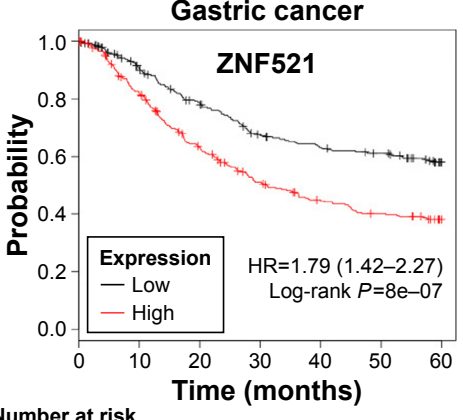

Number at risk

$\begin{array}{llllllll}\text { Low } & 283 & 238 & 201 & 168 & 154 & 146 & 119\end{array}$

$\begin{array}{llllllll}\text { High } & 348 & 275 & 202 & 158 & 134 & 119 & 103\end{array}$
C

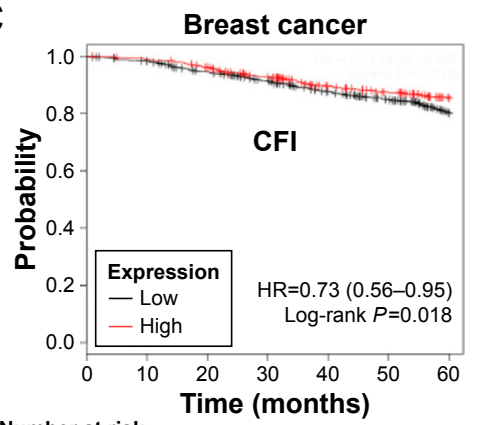

Number at risk

Low $\quad \begin{array}{lllllll}702 & 682 & 645 & 605 & 563 & 527 & 465\end{array}$ $\begin{array}{llllllll}\text { High } & 700 & 690 & 666 & 630 & 584 & 556 & 515\end{array}$

$\mathbf{F}$

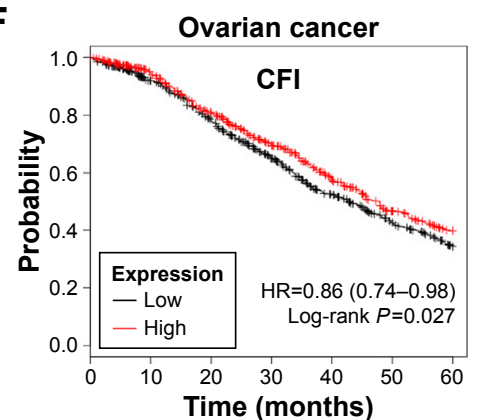

Number at risk

$\begin{array}{llllllll}\text { Low } & 828 & 698 & 566 & 420 & 299 & 209 & 148\end{array}$ High $\quad 828 \quad 724 \quad 584 \quad 447 \quad 323 \quad 219 \quad 162$

\section{I}

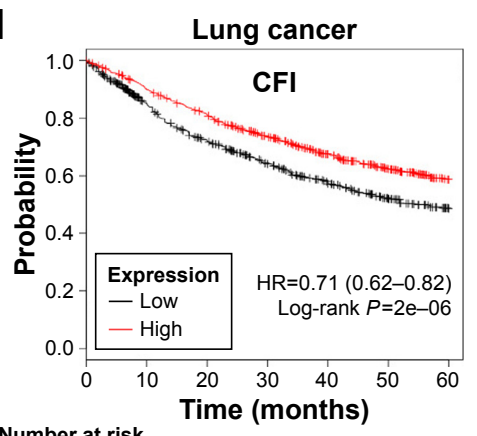

$\begin{array}{llllllll}\text { Low } & 964 & 753 & 626 & 527 & 441 & 363 & 292\end{array}$ $\begin{array}{llllllll}\text { High } & 962 & 857 & 766 & 664 & 557 & 465 & 380\end{array}$

L

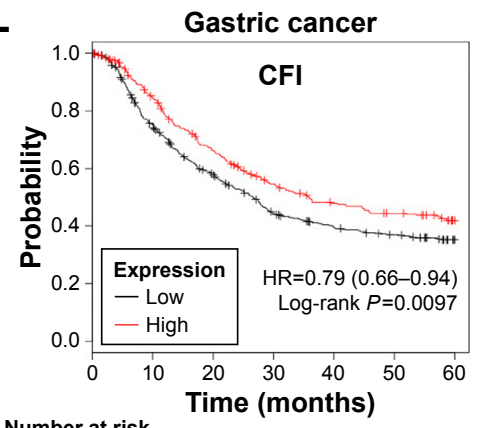

Number at risk

$\begin{array}{llllllll}\text { Low } & 539 & 392 & 293 & 217 & 188 & 171 & 143\end{array}$ High $\quad 337 \quad 271 \quad 208 \quad 165 \quad 139 \quad 127 \quad 107$

Figure 3 The three hub of the survival analysis for cancer datasets by Kaplan-Meier.

Notes: (A-C) Hub genes/proteins (PODN, ZNF52I, CFI) of the survival analysis for breast cancer dataset by Kaplan-Meier. (D-F) Hub genes/proteins (PODN, ZNF52I, $\mathrm{CFI}$ ) of the survival analysis for ovarian cancer dataset by Kaplan-Meier. (G-I) Hub genes/proteins (PODN, ZNF52I, CFI) of the survival analysis for lung cancer dataset by Kaplan-Meier. (J-L) Hub genes/proteins (PODN, ZNF52I, CFI) of the survival analysis for gastric cancer dataset by Kaplan-Meier.

Abbreviation: HR, hazard ratio. 


\section{Acknowledgment}

The authors would like to thank Hai-Ying Ma for his useful suggestions during this work.

\section{Author contributions}

All authors contributed to data analysis, drafting and revising the article, gave final approval of the version to be published, and agree to be accountable for all aspects of the work.

\section{Disclosure}

The authors report no conflicts of interest in this work.

\section{References}

1. Han X, Li X, Zhong G, Liu Z. Regulation of osteogenic differentiation by DNA methylation of the dishevelled gene in bone marrow mesenchymal stem cells. Am J Transl Res. 2017;9(11):4848-4855.

2. Zuk PA, Zhu M, Mizuno H, et al. Multilineage cells from human adipose tissue: implications for cell-based therapies. Tissue Eng. 2001;7(2): 211-228.

3. Mead B, Amaral J, Tomarev S. Mesenchymal stem cell-derived small extracellular vesicles promote neuroprotection in rodent models of glaucoma. Invest Ophthalmol Vis Sci. 2018;59(2):702-714.

4. Brennan MA, Renaud A, Guilloton F, et al. Inferior in vivo osteogenesis and superior angiogeneis of human adipose tissue: a comparison with bone marrow-derived stromal stem cells cultured in xeno-free conditions. Stem Cells Transl Med. 2017;6(12):2160-2172.

5. Börger V, Bremer M, Ferrer-Tur R, et al. Mesenchymal stem/stromal cell-derived extracellular vesicles and their potential as novel immunomodulatory therapeutic agents. Int J Mol Sci. 2017;18(7):1450.

6. Sun Y, Wang Y, Zhou L, et al. Spheroid-cultured human umbilical cordderived mesenchymal stem cells attenuate hepatic ischemia-reperfusion injury in rats. Sci Rep. 2018;8(1):2518.

7. Cai M, Shen R, Song L, et al. Erratum: bone marrow mesenchymal stem cells (BM-MSCs) improve heart function in swine myocardial infarction model through paracrine effects. Sci Rep. 2016;6:31528.

8. Fang X, Neyrinck AP, Matthay MA, Lee JW. Allogeneic human mesenchymal stem cells restore epithelial protein permeability in cultured human alveolar type II cells by secretion of angiopoietin-1.J Biol Chem. 2010;285(34):26211-26222.

9. Baglio SR, Rooijers K, Koppers-Lalic D, et al. Human bone marrowand adipose-mesenchymal stem cells secrete exosomes enriched in distinctive miRNA and tRNA species. Stem Cell Res Ther. 2015;6:127.

10. Valadi H, Ekström K, Bossios A, Sjöstrand M, Lee JJ, Lötvall JO. Exosome-mediated transfer of mRNAs and microRNAs is a novel mechanism of genetic exchange between cells. Nat Cell Biol. 2007; 9(6):654-659.

11. Kosaka N, Iguchi H, Yoshioka Y, Takeshita F, Matsuki Y, Ochiya T. Secretory mechanisms and intercellular transfer of microRNAs in living cells. J Biol Chem. 2010;285(23):17442-17452.

12. Camussi G, Deregibus MC, Bruno S, Grange C, Fonsato V, Tetta C. Exosome/microvesicle-mediated epigenetic reprogramming of cells. Am J Cancer Res. 2011;1(1):98-110.

13. Kosaka N, Iguchi H, Yoshioka Y, Hagiwara K, Takeshita F, Ochiya T. Competitive interactions of cancer cells and normal cells via secretory microRNAs. J Biol Chem. 2012;287(2):1397-1405.
14. Lai RC, Chen TS, Lim SK. Mesenchymal stem cell exosome: a novel stem cell-based therapy for cardiovascular disease. Regen Med. 2011;6(4):481-492.

15. Komaki M, Numata Y, Morioka C, et al. Exosomes of human placentaderived mesenchymal stem cells stimulate angiogenesis. Stem Cell Res Ther. 2017;8(1):219.

16. Sdrimas K, Kourembanas S. MSC microvesicles for the treatment of lung disease: a new paradigm for cell-free therapy. Antioxid Redox Signal. 2014;21(13):1905-1915.

17. Tomasoni S, Longaretti L, Rota C, et al. Transfer of growth factor receptor mRNA via exosomes unravels the regenerative effect of mesenchymal stem cells. Stem Cells Dev. 2013;22(5):772-780.

18. Reis LA, Borges FT, Simões MJ, Borges AA, Sinigaglia-Coimbra R, Schor N. Bone marrow-derived mesenchymal stem cells repaired but did not prevent gentamicin-induced acute kidney injury through paracrine effects in rats. PLoS One. 2012;7(9):e44092.

19. Pascucci L, Coccè V, Bonomi A, et al. Paclitaxel is incorporated by mesenchymal stromal cells and released in exosomes that inhibit in vitro tumor growth: a new approach for drug delivery.J Control Release. 2014; 192:262-270.

20. Torre LA, Bray F, Siegel RL, Ferlay J, Lortet-Tieulent J, Jemal A. Global cancer statistics, 2012. CA Cancer J Clin. 2015;65(2):87-108.

21. Pavlopoulou A, Spandidos DA, Michalopoulos I. Human cancer databases (review). Oncol Rep. 2015;33(1):3-18.

22. Lin E, Cao T, Nagrath S, King MR. Circulating tumor cells: diagnostic and therapeutic applications. Аппи Rev Biomed Eng. 2018;20: 329-352.

23. Beach A, Zhang HG, Ratajczak MZ, Kakar SS. Exosomes: an overview of biogenesis, composition and role in ovarian cancer. J Ovarian Res. 2014;7:14

24. Greening DW, Gopal SK, Xu R, Simpson RJ, Chen W. Exosomes and their roles in immune regulation and cancer. Semin Cell Dev Biol. 2015;40:72-81.

25. Lowry MC, Gallagher WM, O'Driscoll L. The role of exosomes in breast cancer. Clin Chem. 2015;61(12):1457-1465.

26. Azmi AS, Bao B, Sarkar FH. Exosomes in cancer development, metastasis, and drug resistance: a comprehensive review. Cancer Metastasis Rev. 2013;32(3-4):623-642.

27. Cheng L, Sun X, Scicluna BJ, Coleman BM, Hill AF. Characterization and deep sequencing analysis of exosomal and non-exosomal miRNA in human urine. Kidney Int. 2014;86(2):433-444.

28. Huang X, Yuan T, Tschannen M, et al. Characterization of human plasma-derived exosomal RNAs by deep sequencing. BMC Genomics. 2013;14:319.

29. Shimizu-Hirota R, Sasamura H, Kuroda M, Kobayashi E, Saruta T. Functional characterization of podocan, a member of a new class in the small leucine-rich repeat protein family. FEBS Lett. 2004;563(1-3): 69-74.

30. Mega T, Lupia M, Amodio N, et al. Zinc finger protein 521 antagonizes early B-cell factor 1 and modulates the B-lymphoid differentiation of primary hematopoietic progenitors. Cell Cycle. 2011;10(13):2129-2139.

31. Shen S, Pu J, Lang B, Mccaig CD. A zinc finger protein Zfp521 directs neural differentiation and beyond. Stem Cell Res Ther. 2011;2(2):20.

32. Mesuraca M, Chiarella E, Scicchitano S, et al. ZNF423 and ZNF521: EBF1 antagonists of potential relevance in B-lymphoid malignancies. Biomed Res Int. 2015;2015:165238-165239.

33. Okroj M, Holmquist E, Nilsson E, Anagnostaki L, Jirström K, Blom AM. Local expression of complement factor I in breast cancer cells correlates with poor survival and recurrence. Cancer Immunol Immunother. 2015;64(4):467-478. 


\section{Publish your work in this journal}

OncoTargets and Therapy is an international, peer-reviewed, open access journal focusing on the pathological basis of all cancers, potential targets for therapy and treatment protocols employed to improve the management of cancer patients. The journal also focuses on the impact of management programs and new therapeutic agents and protocols on

patient perspectives such as quality of life, adherence and satisfaction. The manuscript management system is completely online and includes a very quick and fair peer-review system, which is all easy to use. Visit http://www.dovepress.com/testimonials.php to read real quotes from published authors.

Submit your manuscript here: http://www.dovepress.com/oncotargets-and-therapy-journal 